\title{
Modeling and Analysis of Paste Freezing in Freeze-Form Extrusion Fabrication of Thin-Wall Parts via a Lumped Method
}

\author{
Mingyang Li ${ }^{*}$, Amir Ghazanfari, Wenbin Li, Robert G. Landers, Ming C. Leu \\ Department of Mechanical and Aerospace Engineering, Missouri University of Science and \\ Technology, Rolla, Missouri 65409 \\ E-mail addresses: m189c@mst.edu (M. Li) ag4nc@mst.edu (A. Ghazanfari)w19ff@mst.edu \\ (W. Li) landersr@mst.edu (R. G. Landers) mleu@mst.edu (M. C. Leu) \\ * Corresponding author.
}

\section{Abstract}

During the Freeze-form Extrusion Fabrication (FEF) process using aqueous-based pastes, the freezing environment solidifies the water content in the paste and thus aids the part in maintaining its shape. Because of the small temperature variation in the paste and the paste's large latent heat introduced by the water contained in the paste, the paste typically freezes slowly during the FEF process. In this study, a lumped method was used to model the solidification process of thin-wall parts fabricated in a layer-by-layer manner. A non-dimensional analytical solution for the freezing time of parts with large numbers of layers was obtained using the lumped method, and its corresponding dimensional solution was compared with numerical simulation results. Based on the analytical solution, the effects of two non-dimensional factors (i.e., non-dimensional latent heat and effective Biot number) and six dimensional factors (i.e., convection coefficient, paste material, paste solids loading, ambient temperature, filament height, and filament width) on the freezing time were investigated. Experiments using different solids loadings pastes and extrusion parameters were conducted to validate the freezing time predictions generated by the lumped method. Possible error sources during the experiments were 
discussed and their effects were estimated. The results show that the lumped method can be used to accurately predict the freezing time of thin-wall parts within $17.9 \%$ differences from the measured results.

Keywords: freeze-form extrusion fabrication, paste solidification, analytical solution, experimental validation.

\section{Nomenclature}

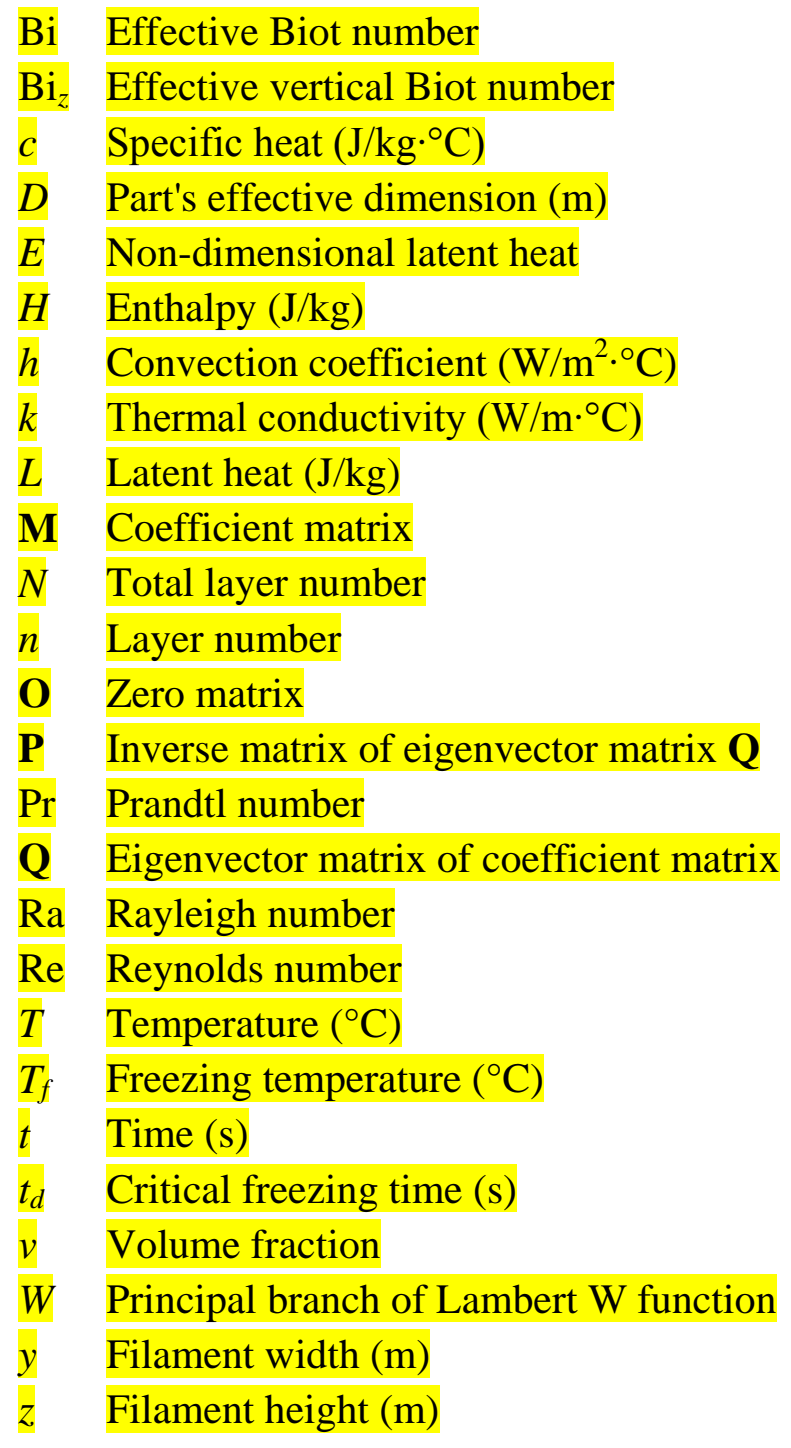


$\eta \quad$ Non-dimensional enthalpy

$\lambda \quad$ Eigenvalue of coefficient matrix

$\rho$ Density $\left(\mathrm{kg} / \mathrm{m}^{3}\right)$

$\tau \quad$ Non-dimensional time

$\tau_{d} \quad$ Non-dimensional critical freezing time

$\chi \quad$ Coefficient of volume expansion $(1 / \mathrm{K})$

\section{Introduction}

Temperature and heat transfer rate are important process parameters in many Additive Manufacturing (AM) processes. They typically have a direct impact on many properties of the fabricated parts. Therefore, thermal analysis is widely used to understand the physics of AM processes and to improve the part quality of these processes. Zeng et al. (2012) reviewed the thermal analysis methods in Laser Sintering and Selective Laser Melting processes. The governing equations, boundary conditions, material properties, energy source characteristics, analytical solutions, and numerical simulations were reviewed in that paper. Tapia and Elwany (2014) reviewed temperature and displacement sensors, monitoring setup, and control research progress in metal-based AM processes. Both of the review papers noted that there is still a significant lack of physical understanding and mathematical models for thermal analysis of AM processes. An efficient analytical or numerical method would be beneficial for the optimization of process parameters and the improvement of part quality. In recent research studies, numerical simulation was the main method used to conduct thermal analysis in AM processes. Denlinger et al. (2014) used adaptive meshing method and Michaleris (2014) used dimension reduction in simulations to reduce node number and simulation time. Although simulations are becoming more efficient, they are still computationally expensive even for simple geometries. And the basic physical understanding of layer-by-layer solidification process is still lacking. 
Huang et al. (2015) reviewed the current state of AM processes and stated that Extrusion Free-Forming (EFF) processes constitute an important part of AM processes. Similar to other AM processes, initial material temperature, building environment temperature, and heat transfer conditions significantly affect the final product quality in the EFF processes. Huang et al. (2006) introduced the Freeze-form Extrusion Fabrication (FEF) process, in which the temperature's effect is even more significant. As an EFF process, FEF uses aqueous-based ceramic pastes as the raw material, and uses ram extruders to force out the paste filament into a low temperature environment, which freezes the water present in the paste, thereby helping to maintain the part's shape. Because of the small temperature variation in the paste and the paste's large latent heat due to the water in the paste, the paste typically freezes slowly during the part fabrication process. If the freezing is not complete before building the next layer, the part being fabricated may deform or even collapse. For successfully built parts, their dimensional accuracy and surface finish are also strongly affected by part's freezing time.

In a previous study, Li et al. (2014) used numerical simulation to investigate the solidification time of thin-wall parts during the FEF process with respect to seven factors. That research showed that the paste freezing time is very short when the paste is deposited near the substrate, then increases as the part height increases, and finally reaches a steady state. The relationship between steady-state freezing time and total time between layers was also studied in that research. Although the numerical simulation study had considered a large number of factors, some factors affecting the solidification process were not considered, and the solidification mechanism of the layer-by-layer paste extrusion processes was not identified.

Due to the complexity of AM processes, most analytical analyses focus on single-track deposition. However, the basic fabrication unit (one layer) in AM processes is so small that one 
part typically requires tens to thousands of layers. With such a large number of layers, the boundary conditions of each layer being fabricated will be very different from the simple assumptions used in typical analytical analyses. Thus, the understanding of the fabrication of a single track cannot be extended to the fabrication of multiple layers. On the other hand, a large number of layers also means the fabrication unit (i.e., one layer) is relatively small compared to the entire part. Therefore, it is possible to ignore the variations inside each layer, and to focus more on the interaction between layers. Similarly, it is possible to ignore the transient condition for a small number of layers and to focus on the steady state condition for a large number of layers.

In this paper, a lumped method was used to analyze FEF processes for fabricating thin-wall parts in a layer-by-layer manner. A non-dimensional analytical solution for the critical freezing time (i.e., when the steady-state freezing time is equal to the total time between layers) was obtained from this method, and its corresponding dimensional solution was compared with numerical simulation results. Experiments using different pastes and extrusion parameters were conducted to validate the predictions using the lumped method. Possible error sources during the experiments were discussed and their corresponding effects were estimated. The model shows that the non-dimensional analytical solution is mainly affected by two non-dimensional factors, i.e., non-dimensional latent heat and effective Biot number. Each of the two non-dimensional factors may be affected by multiple dimensional factors including convection coefficient, paste material properties, paste solids loading, ambient temperature, filament height, and filament width, whose effects on the critical freezing time were also investigated. Experimental results show that the lumped method can be used to predict the critical freezing times of thin-wall parts within $17.9 \%$ differences from the measured results. 


\section{Theory}

A lumped method is used in this paper to study the temperature change and the freezing time of a thin-wall part during the FEF process. The governing energy equation is developed by considering the temperature gradient within each layer to be negligible. Then, by nondimensionalizing and solving the governing energy equation, a non-dimensional analytical solution for the paste freezing time is obtained. Further, the estimations of thermal conductivity and convection coefficients as the connections between experiment and dimensional factors are also discussed.

\subsection{Lumped Method}

Consider a thin wall composed of $N$ paste filaments, as shown in Figure 1. Each filament has width $y(\mathrm{~m})$ and height $z(\mathrm{~m})$. According to Fourier's Law, the governing energy equation for the $n^{\text {th }}$ filament is

$$
\frac{d H_{n}(t)}{d t}=-\frac{k}{\rho z^{2}}\left[T_{n}(t)-T_{n-1}(t)\right]+\frac{k}{\rho z^{2}}\left[T_{n+1}(t)-T_{n}(t)\right]-\frac{2 h}{\rho y}\left[T_{n}(t)-T_{0}\right]
$$

where $H_{n}(\mathrm{~J} / \mathrm{kg})$ and $T_{n}\left({ }^{\circ} \mathrm{C}\right)$ are the enthalpy and temperature, respectively, of the $n^{\text {th }}$ filament, $T_{0}$ $\left({ }^{\circ} \mathrm{C}\right)$ is the ambient temperature, and $t(\mathrm{~s}), k\left(\mathrm{~W} / \mathrm{m} \cdot{ }^{\circ} \mathrm{C}\right), \rho\left(\mathrm{kg} / \mathrm{m}^{3}\right), c\left(\mathrm{~J} / \mathrm{kg} \cdot{ }^{\circ} \mathrm{C}\right)$, and $h\left(\mathrm{~W} / \mathrm{m}^{2} \cdot{ }^{\circ} \mathrm{C}\right)$ are time, thermal conductivity, density, specific heat, and convection coefficient, respectively. When $n=1, T_{n-1}$ is the substrate temperature, which is $T_{0}$. When $n=N$, the conduction term $k\left[T_{n+1}(t)-T_{n}(t)\right] / \rho z^{2}$ is replaced by the convection term $-h\left[T_{n}(t)-T_{0}\right] / \rho y$. 


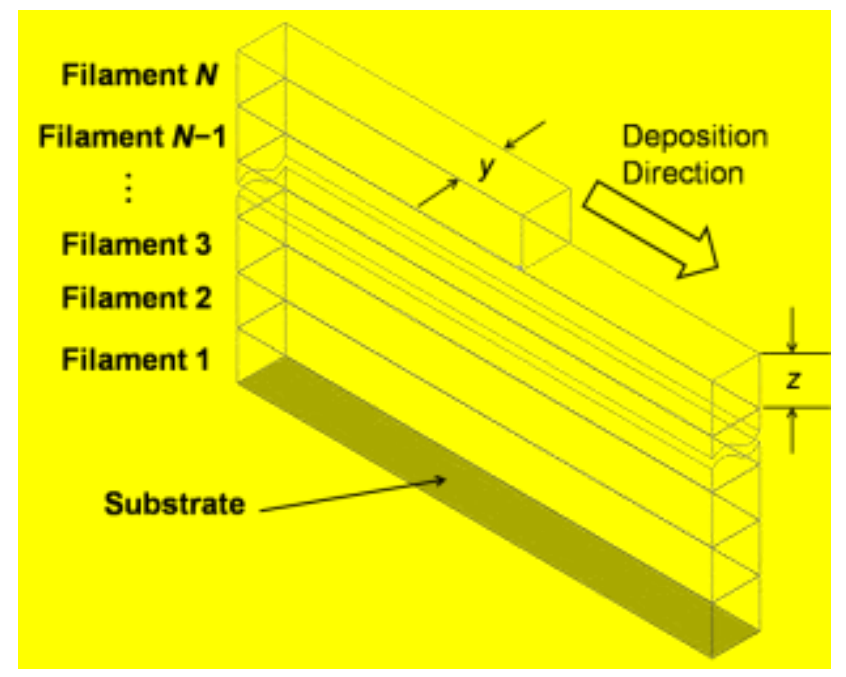

Figure 1. Schematic of a thin wall composed of $N$ paste filaments.

A thin wall may contain three regions: solid, mushy, and liquid. Let subscripts $s, m$, and $l$ denote the solid, mushy, and liquid regions, respectively. Considering the specific heat to be constant, the temperature can be obtained based on the definition of enthalpy

$$
T(H)=\left\{\begin{array}{cc}
\frac{H}{c}+T_{0} & H \leq c T_{f} \\
T_{f} & c T_{f}<H<c T_{f}+L \\
\frac{H-L}{c}+T_{0} & H \geq c T_{f}+L
\end{array}\right.
$$

where $T_{f}\left({ }^{\circ} \mathrm{C}\right)$ is the freezing temperature and $L(\mathrm{~J} / \mathrm{kg})$ is the latent heat. Let $\tau=k t / \rho c z^{2}, \mathrm{Bi}=$ $h z^{2} / k y, \mathrm{Bi}_{z}=h z / k, \eta=H / c\left(T_{f}-T_{0}\right)$, and $E=L / c\left(T_{f}-T_{0}\right)$ denote non-dimensional time, effective Biot number, effective vertical Biot number, non-dimensional enthalpy, and non-dimensional latent heat, respectively. Combining the governing equations for each layer and rewriting them in matrix form

$$
\left[\begin{array}{c}
\boldsymbol{\eta}_{s}^{\prime}(\tau) \\
\boldsymbol{\eta}_{m}^{\prime}(\tau) \\
\boldsymbol{\eta}_{l}^{\prime}(\tau)
\end{array}\right]=\left[\begin{array}{ccc}
\mathbf{M}_{s} & \mathbf{O} & \mathbf{O} \\
\mathbf{U} & \mathbf{O} & \mathbf{D} \\
\mathbf{O} & \mathbf{O} & \mathbf{M}_{l}
\end{array}\right]\left[\begin{array}{l}
\boldsymbol{\eta}_{s}(\tau) \\
\boldsymbol{\eta}_{m}(\tau) \\
\boldsymbol{\eta}_{l}(\tau)
\end{array}\right]+\left[\begin{array}{c}
\boldsymbol{\beta}_{s} \\
\boldsymbol{\beta}_{m} \\
\boldsymbol{\beta}_{l}
\end{array}\right]
$$


where $\boldsymbol{\eta}_{*}^{\prime}(\tau)$ is the derivative of $\boldsymbol{\eta}_{*}(\tau)$ with respect to $\tau$, and the subscript $*$ is $s$ for the solid region, $m$ for the mushy region, and $l$ for the liquid region. The explicit forms of $\mathbf{M} * \mathbf{U}, \mathbf{D}$, and $\boldsymbol{\beta} *$ are given in Eqs. (A2)-(A8) in the Appendix.

When a new layer is deposited, the freezing time of the layer is composed of two parts. The first part is the time spent for the liquid region to transform into a mushy region. The second part is the time spent for the mushy region to transform into a solid region. Due to the fact that the latent heat of paste is much larger than the specific heat of paste and the initial temperature is very close to the freezing temperature, the liquid region transforms into a mushy region much faster than the mushy region transforms into a solid region. Therefore, the freezing time is approximated by the summation of the non-dimensional times required for each layer in the mushy region to transform into a solid layer

$$
t_{f}=\frac{\rho c z^{2}}{k} \sum_{n=1}^{N_{m}} \tau_{f, n}
$$

where $\tau_{f, n}$ is the non-dimensional time for the $n^{\text {th }}$ mushy layer to transform into a solid layer. This non-dimensional time is obtained by solving

$$
\eta_{m, n}\left(\tau_{f, n}\right)=1 \quad\left(n=1,2, \ldots, N_{m}\right)
$$

To prevent the part from deforming or collapsing during the fabrication process, the new layer should only be deposited when the previous layers are frozen. In that case, the mushy region typically only consists of one layer. The explicit form of Eq. (5) is derived from Eqs. (A1)-(A26) in the Appendix. Noting $\eta_{m, 1}(0) \approx 1+E$ and solving Eq. (5) as shown by Eqs. (A26)-(A42) in the Appendix, the non-dimensional critical freezing time is 


$$
\tau_{d}=\tau_{f, 1}=\frac{E-C_{\lambda 2}-C_{\eta}}{1+2 \mathrm{Bi}+C_{\lambda 1}}
$$

where

$$
\begin{gathered}
C_{\lambda 1}(\mathrm{Bi})=\lim _{N_{s} \rightarrow \infty}\left\{\frac{4}{2 N_{s}+1} \sum_{i=1}^{N_{s}}\left[\frac{1}{\lambda_{s, i}} \sin ^{2}\left(\frac{2 i \pi}{2 N_{s}+1}\right)\right]\right\} \\
C_{\lambda 2}(\mathrm{Bi})=\lim _{N_{s} \rightarrow \infty}\left\{\frac{4}{2 N_{s}+1} \sum_{i=1}^{N_{s}}\left[\frac{1}{\lambda_{s, i}^{2}} \sin ^{2}\left(\frac{2 i \pi}{2 N_{s}+1}\right)\right]\right\}=\frac{d C_{\lambda 1}}{2 d \mathrm{Bi}} \\
C_{\eta}\left(\mathrm{Bi}, \tau_{r}\right)=\lim _{N_{s} \rightarrow \infty}\left\{\frac{4}{2 N_{s}+1} \sum_{i=1}^{N_{s}}\left\{\frac{1}{\lambda_{s, i}} \sin \left[\frac{\left(2 N_{s}-1\right) i \pi}{2 N_{s}+1}\right] \sum_{j=1}^{N_{s}}\left\{\sin \left[\frac{i(2 j-1) \pi}{2 N_{s}+1}\right] \eta_{s, j}(0)\right\}\right\}\right\} \\
\lambda_{s, i}=-2\left[1+\mathrm{Bi}-\cos \left(\frac{2 i \pi}{2 N_{s}+1}\right)\right]
\end{gathered}
$$

the subscript $d$ denotes steady state, and the subscript $r$ denotes the total time between layers (i.e., the summation of the deposition time for the current layer and the dwell time between the current and next layers).

\subsection{Paste Material Properties}

The paste used for the experiments conducted in this paper is mainly composed of ceramic particles and water; therefore, the paste's density, specific heat, and latent heat can be computed using the law of mixtures in the previous work by Li et al. (2014). However, the estimation of thermal conductivity is more complex. There are numerous papers studying the thermal conductivity of two-phase mixtures. Brailsford and Major (1964) classified these methods into several different conditions. Due to the high solids loading of the paste used in this study (45-60 vol.\%), it can be assumed that the two phases (i.e., ceramic particles and water) have similar 
distributions. Therefore, the General Effective Medium Theory (GEMT) introduced by Landauer (1952) is used to estimate the paste thermal conductivity

$$
\begin{aligned}
k_{p} & =\operatorname{GEMT}\left(k_{c}, k_{w}, v_{c}, v_{w}\right) \\
& =\frac{\left(3 v_{c}-1\right) k_{c}+\left(3 v_{w}-1\right) k_{w}+\left\{\left[\left(3 v_{c}-1\right) k_{c}+\left(3 v_{w}-1\right) k_{w}\right]^{2}+8 k_{c} k_{w}\right\}^{\frac{1}{2}}}{4}
\end{aligned}
$$

where $k\left(\mathrm{~W} / \mathrm{m} \cdot{ }^{\circ} \mathrm{C}\right)$ is thermal conductivity, $v$ is volume fraction, and the subscripts $p, w$, and $c$ denote paste, water, and ceramic, respectively.

A possible source of error in this calculation is that the trace amount of binder and dispersant may accumulate on the surface of ceramic particles, changing the paste's effective thermal conductivity. The binder and the dispersant have smaller thermal conductivities than the ceramic particles and the water; therefore, a thin binder and dispersant film between the ceramic particles and the water may significantly reduce the paste thermal conductivity. Since the binder and the dispersant are assumed to accumulate on the surface of the ceramic particles, they have the same distribution as the ceramic particles and, thus, the effective thermal conductivity of the mixture of ceramic particles, binder, and dispersant is

$$
k_{u}=\operatorname{GEMT}\left(k_{c}, k_{b}, \frac{v_{c}}{v_{c}+v_{b}}, \frac{v_{b}}{v_{c}+v_{b}}\right)
$$

where the subscript $b$ denotes binder plus dispersant. In this case, the paste thermal conductivity is

$$
k_{p}^{*}=\operatorname{GEMT}\left(k_{u}, k_{w}, v_{c}, v_{w}\right)
$$

For the cases studied in this paper, the volume fraction of binder and dispersant together is less than $3 \%$ and it can be shown that the thermal conductivity error (i.e., the difference between $k_{p}$ and $\left.k_{p}^{*}\right)$ is less than $4 \%$ and the critical freezing time error is less than $2 \%$. Since the 
distribution of binder and dispersant can be very complex and the error is small, the effect of binder and dispersant on paste thermal conductivity in this study is neglected. The paste thermal conductivities used in the studies conducted in this paper will be computed using Eq. (11).

\subsection{Convection Coefficient}

Hollow square parts will be fabricated in the experimental study to be described below. One of the faces of the square part fabricated in the experiment will be perpendicular to the air flow. When the convection condition is forced, Cengel (2002) showed the convection coefficient can be estimated by

$$
h=\left(0.102 \operatorname{Re}^{0.675} \operatorname{Pr}^{\frac{1}{3}}\right) \frac{k_{0}}{D}
$$

where $D(\mathrm{~m})$ is the part's effective dimension, which is the side length for square parts, $R e$ is the Reynolds number for air, and $\operatorname{Pr}$ is the Prandtl number for air. When the convection condition is natural, Cengel (2002) showed the convection coefficient can be estimated by

$$
h=\left\{0.825+\frac{0.387 \mathrm{Ra}^{1 / 6}}{\left[1+(0.492 / \mathrm{Pr})^{9 / 16}\right]^{8 / 27}}\right\} \frac{k_{0}}{D}
$$

where the part's effective dimension $D$ is the part height under natural convection, $k_{0}$ is the thermal conductivity of air, and $R a$ is the Rayleigh number

$$
\mathrm{Ra}=\frac{g \chi\left(T-T_{0}\right) D^{3}}{v_{0}^{2}} \operatorname{Pr}
$$

where $\chi(1 / \mathrm{K})$ is the coefficient of volume expansion, which is considered to be $1 /(T+273.15)$ for air, $g\left(\mathrm{~m} / \mathrm{s}^{2}\right)$ is the gravitational constant, and $v_{0}\left(\mathrm{~m}^{2} / \mathrm{s}\right)$ is the kinematic viscosity of air. 


\section{Analytical Results and Discussion}

According to Eqs.(6)-(10), the non-dimensional critical freezing time is a function of the non-dimensional latent heat and effective Biot number. Although the non-dimensional total time between layers is only presented in Eq. (9), it is solved by combining Eqs.(3) and (6). Therefore, a random initial enthalpy distribution can be fed into Eq. (3) with a reasonable layer number, which is typically between 50 and 150 . After several iterations of Eq. (3), the critical freezing time is then obtained from Eq. (6).

\subsection{Lumped Method versus Numerical Simulation}

The critical freezing time computed using the lumped method are compared in Figure 2 to the numerical simulations results generated using the method described by Li et al. (2014). The material properties used in the computation are listed in Table 1 for zirconia $\left(\mathrm{ZrO}_{2}\right)$, zirconium carbide $(\mathrm{ZrC})$, alumina $\left(\mathrm{Al}_{2} \mathrm{O}_{3}\right)$, and tungsten $(\mathrm{W})$. The convection coefficient was $35 \mathrm{~W} / \mathrm{m}^{2} \cdot{ }^{\circ} \mathrm{C}$, the initial paste temperature was $5{ }^{\circ} \mathrm{C}$, the ambient temperature was $-10{ }^{\circ} \mathrm{C}$, and the filament height and width were both $580 \mu \mathrm{m}$. As the Biot number increases, the percent errors for the data points are $2.04 \%, 3.25 \%, 6.29 \%, 14.4 \%$, respectively. The error is mainly introduced by neglecting the temperature gradient inside a single filament in the lumped method. As the Biot number increases, the temperature gradient inside a single filament becomes larger. Therefore, the heat transfer rate computed by the numerical simulation is larger than the heat transfer rate assumed in the lumped method, causing the critical freezing time computed by the numerical simulation to be less than the critical freezing time computed by the lumped method. It was shown by Cengel (2002) that the lumped system analysis is generally applicable if the Biot number is smaller than 0.1 , as the error will remain within 5\%. However, in AM processes, the 
error will be amplified by the layer-by-layer manner of the fabrication process. The lumped method can be successfully employed when the effective Biot number is smaller than 0.004 since the error will remain within approximately $7 \%$, which is the magnitude of the experimental errors observed in the tests conducted in Section 4.3. In the cases studied in this paper, the convection coefficient is typically smaller than $35 \mathrm{~W} / \mathrm{m}^{2} \cdot{ }^{\circ} \mathrm{C}$ and the paste conductivity is typically larger than $6.0 \mathrm{~W} / \mathrm{m} \cdot{ }^{\circ} \mathrm{C}$; therefore, the effective Biot numbers are smaller than 0.004 . The convergence history of the computations is shown in Figure 3. All of the computations started with the following initial condition: 150 layers have been deposited and their enthalpies are linearly distributed from zero to the solidus enthalpy value along $z$ direction. All of the computations reached steady state after an additional 20 layers were deposited.

Table 1. Properties of pastes with $45 \%$ solids loading used for simulations in Figure 2.

\begin{tabular}{ccccc}
\hline Paste material & $\mathrm{ZrO}_{2}$ & $\mathrm{ZrC}$ & $\mathrm{Al}_{2} \mathrm{O}_{3}$ & $\mathrm{~W}$ \\
\hline $\begin{array}{c}\text { Density }\left(\mathrm{kg} / \mathrm{m}^{3}\right) \\
\text { Average thermal }\end{array}$ & 3025 & 3579 & 2350 & 9235 \\
$\begin{array}{c}\text { conductivity }\left(\mathrm{W} / \mathrm{m} \cdot{ }^{\circ} \mathrm{C}\right) \\
\text { Average specific heat } \\
\left(\mathrm{J} / \mathrm{kg} \cdot{ }^{\circ} \mathrm{C}\right)\end{array}$ & 1.8 & 6.0 & 20.0 & 78.6 \\
\begin{tabular}{c} 
Latent heat $\left(10^{4} \mathrm{~J} / \mathrm{kg}\right)$ \\
\hline
\end{tabular} & 6.07 & 5.13 & 7.82 & 1.99 \\
\hline
\end{tabular}




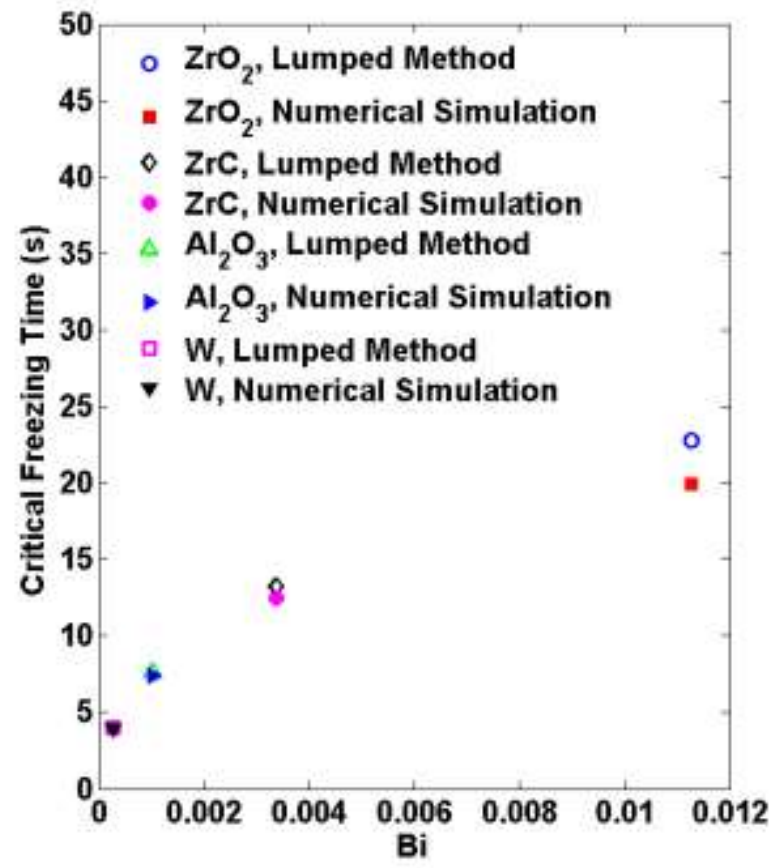

Figure 2. Critical freezing time computed by lumped method and numerical simulation.

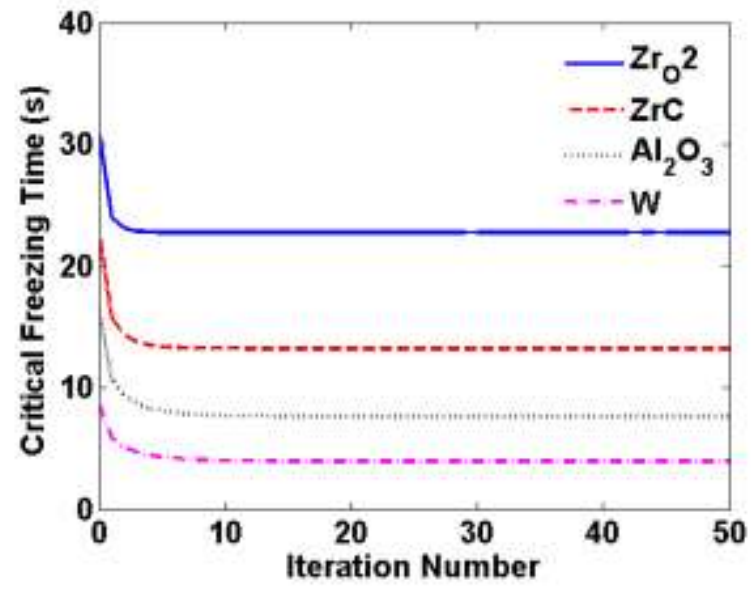

Figure 3. Convergence history of the critical freezing times computed using the lumped method. 


\subsection{Effects of Non-dimensional Factors on Critical Freezing Time}

As seen in Eq. (6), the non-dimensional critical freezing time is a function of the nondimensional latent heat and effective Biot number. For the cases studied in Section 3.1, the nondimensional latent heats are between 5 and 8 , while the effective Biot number varies from $5 \times 10^{-4}$ to $1.5 \times 10^{-2}$. Therefore, the studies conducted in this paper will focus on the ranges $E \in[4,9]$ and $\mathrm{Bi} \in\left[5 \times 10^{-4}, 2 \times 10^{-2}\right]$

\subsubsection{Non-dimensional latent heat}

To study the effect of non-dimensional latent heat, differentiating Eq. (6) with respect to nondimensional latent heat and rearranging

$$
\frac{\partial \tau_{d}}{\partial E}=\frac{1}{1+2 \mathrm{Bi}+C_{\lambda 1}+\frac{\partial C_{\eta}}{\partial \tau_{d}}}
$$

The term $\partial C_{\eta} / \partial \tau_{d}$ is difficult to express explicitly. The iteratively computed value of $\partial C_{\eta} / \partial \tau_{d}$ versus $\tau_{d}$ is plotted in Figure 4. In the range of effective Biot numbers considered in Figure 4, the value of $\partial C_{\eta} / \partial \tau_{d}$ is negligible compared to the term $1+2 \mathrm{Bi}+C_{\lambda 1}$. Therefore, the relationship between the non-dimensional critical freezing time and the non-dimensional latent heat is nearly linear, and the slope is a function of the effective Biot number, as plotted in Figure 5. The smaller the effective Biot number, the more rapidly the non-dimensional critical freezing time changes with respect to non-dimensional latent heat. 


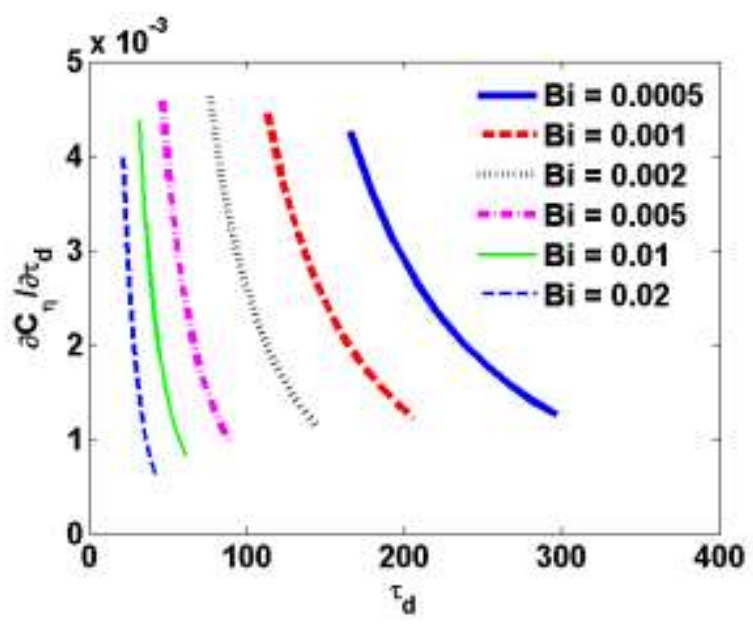

Figure 4. Computed $\partial C_{\eta} / \partial \tau_{d}$ as a function of non-dimensional critical freezing time.

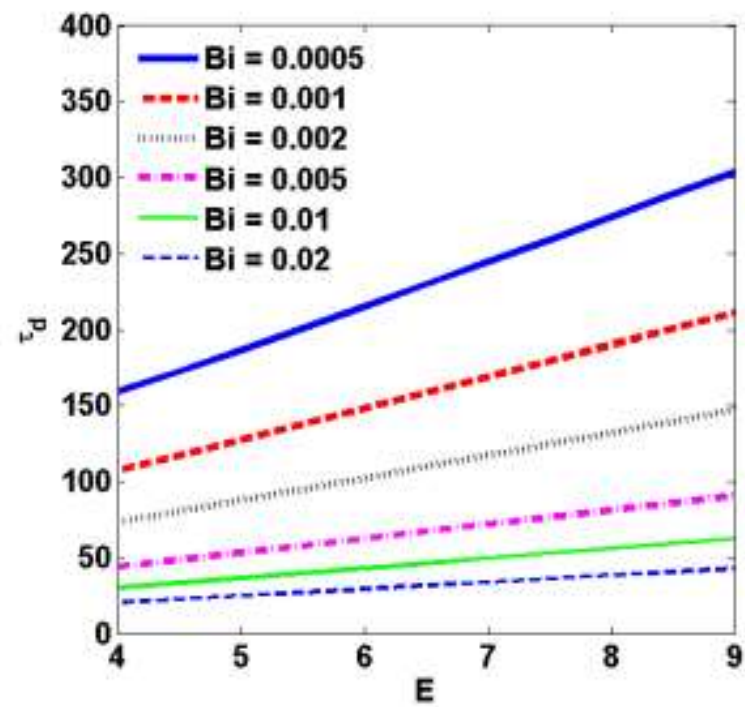

Figure 5. Non-dimensional critical freezing time as a function of non-dimensional latent heat for various effective Biot numbers.

\subsubsection{Effective Biot number}

To study the effect of the effective Biot number, differentiating Eq. (6) with respect to the effective Biot number and rearranging 


$$
\frac{\partial \tau_{d}}{\partial \mathrm{Bi}}=-\frac{\left(\frac{d C_{\lambda 2}}{d \mathrm{Bi}}+\frac{\partial C_{\eta}}{\partial \mathrm{Bi}}\right)}{\left(1+2 \mathrm{Bi}+C_{\lambda 1}\right)}-\frac{\left(E-C_{\lambda 2}-C_{\eta}\right)\left(2+2 C_{\lambda 2}\right)}{\left(1+2 \mathrm{Bi}+C_{\lambda 1}\right)^{2}}
$$

which is also a complex function that is difficult to express explicitly. The terms $C_{\lambda 1}, C_{\lambda 2}$, and $C_{\eta}$, are plotted versus effective Biot number in Figures 6 and 7. All of these terms are highly nonlinear, and they all can be treated as functions of the effective Biot number. The partial derivative of the non-dimensional critical freezing and the non-dimensional critical freezing time itself are plotted vs. effective Biot number in Figures 8 and 9, respectively. The partial derivative of the non-dimensional critical freezing time with respect to effective Biot number is always negative. When the effective Biot number is small, the magnitude of the partial derivative of the critical freezing time to the Biot number is large. As the effective Biot number increases, the magnitude of the partial derivative decreases nonlinearly.

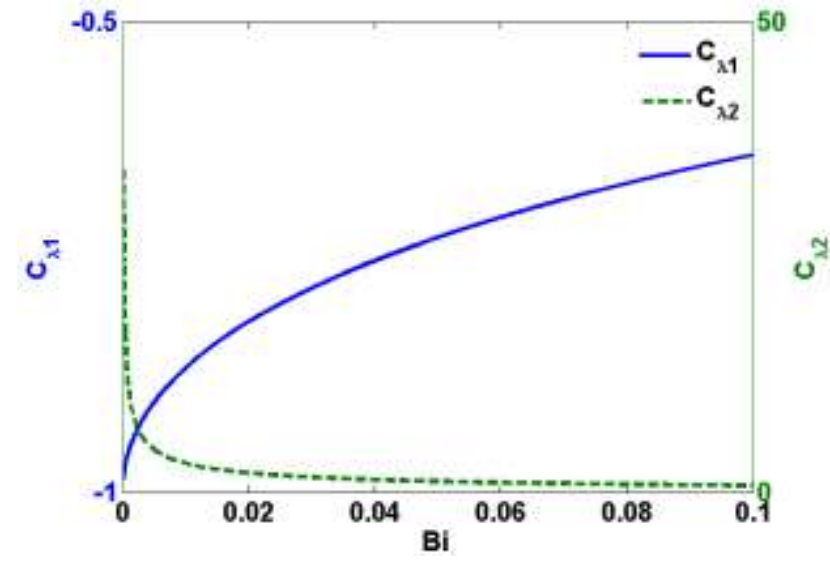

Figure 6. $C_{\lambda 1}$ and $C_{\lambda 2}$ as a function of effective Biot number. 


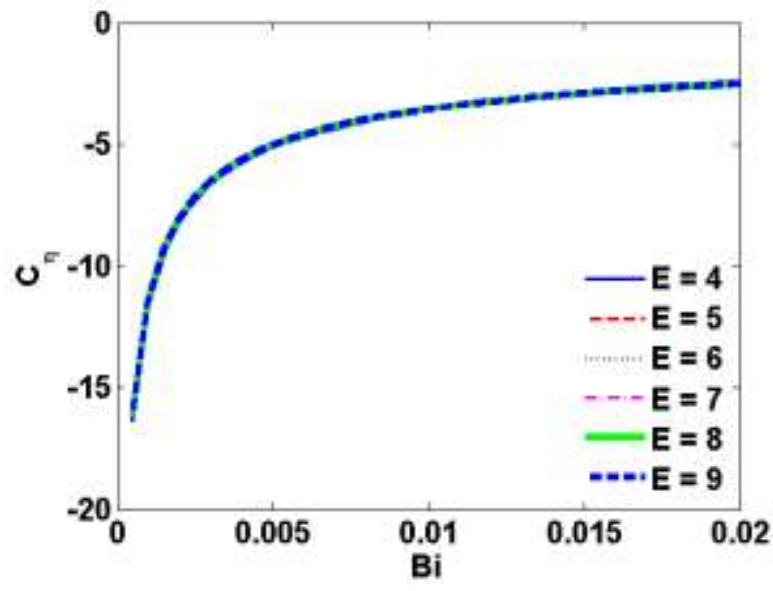

Figure 7. Computed $C_{\eta}$ as a function of effective Biot number.

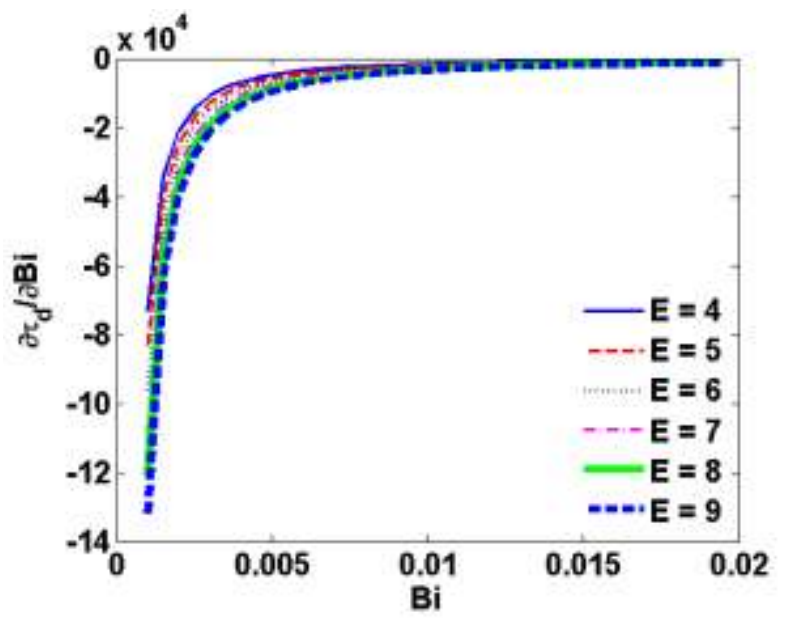

Figure 8. Partial derivative of non-dimensional critical freezing time with respect to effective Biot number. 


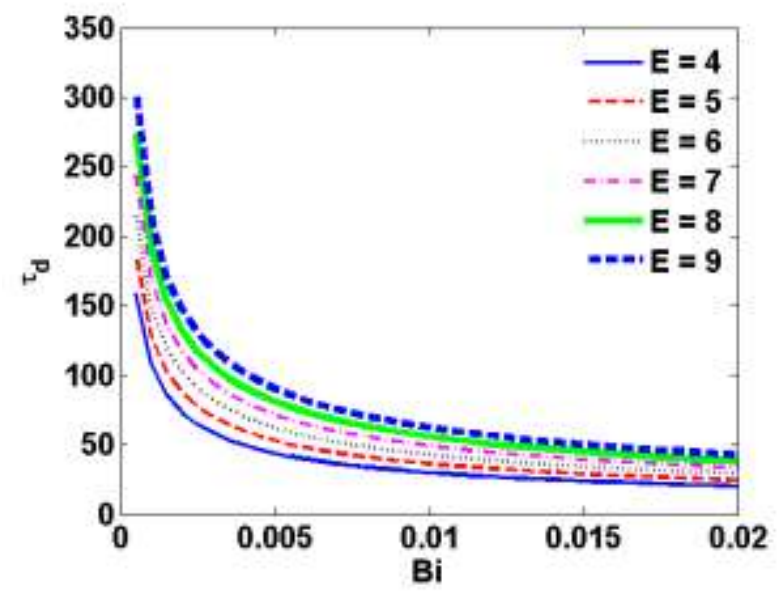

Figure 9. Non-dimensional critical freezing time as a function of effective Biot number.

\subsection{Effects of Dimensional Factors on Critical Freezing Time}

Only two non-dimensional factors can affect the non-dimensional critical freezing time. However, each non-dimensional factor consists of multiple dimensional factors, and some of the dimensional factors can change both non-dimensional factors. The dimensional freezing time $t_{d}$ also involves several dimensional factors after conversion from the non-dimensional freezing time $\tau_{d}$. A discussion of the effects of the dimensional factors is given below.

\subsubsection{Convection coefficient}

To study the effect of convection coefficient, Eq. (6) is differentiated with respect to convection coefficient. The convection coefficient only affects the effective Biot number. Therefore

$$
\frac{\partial t_{d}}{\partial h}=\frac{\rho c z^{2}}{k} \frac{\partial \tau_{d}}{\partial \mathrm{Bi}} \frac{\partial \mathrm{Bi}}{\partial h}=\frac{\rho c z^{4}}{k^{2} y} \frac{\partial \tau_{d}}{\partial \mathrm{Bi}}
$$

For the same paste and environmental conditions, the relationship between critical freezing time and convection coefficient is proportional to the relationship between the non-dimensional 
critical freezing time and the effective Biot number. Therefore, as the convection coefficient increases, the critical freezing time decreases nonlinearly.

\subsubsection{Paste material properties}

The change of paste material will change all of the material properties, i.e., thermal conductivity $(k)$, specific heat $(c)$, latent heat $(L)$, and density $(\rho)$. To study the effect of material properties, Eq. (6) is differentiated with respect to each material property

$$
\begin{gathered}
\frac{\partial t_{d}}{\partial k}=\frac{-\rho c z^{2}}{k^{2}} \tau_{d}+\frac{\rho c z^{2}}{k} \frac{\partial \tau_{d}}{\partial \mathrm{Bi}} \frac{\partial \mathrm{Bi}}{\partial k}=-\frac{\rho c z^{2}}{k^{2}}\left(\tau_{d}+\mathrm{Bi} \frac{\partial \tau_{d}}{\partial \mathrm{Bi}}\right)=-\frac{\rho c z^{2}}{k^{2}} \frac{\partial\left(\tau_{d} \mathrm{Bi}\right)}{\partial \mathrm{Bi}} \\
\frac{\partial t_{d}}{\partial c}=\frac{\rho z^{2}}{k} \tau_{d}+\frac{\rho c z^{2}}{k} \frac{\partial \tau_{d}}{\partial E} \frac{\partial E}{\partial c} \approx \frac{\rho z^{2}}{k}\left[\tau_{d}-\frac{E}{\left(1+2 \mathrm{Bi}+C_{\lambda 1}\right)}\right]=-\frac{\rho z^{2}\left(C_{\lambda 2}+C_{\eta}\right)}{k\left(1+2 \mathrm{Bi}+C_{\lambda 1}\right)} \\
\frac{\partial t_{d}}{\partial L}=\frac{\rho c z^{2}}{k} \frac{\partial \tau_{d}}{\partial E} \frac{\partial E}{\partial L} \approx \frac{\rho z^{2}}{k\left(1+2 \mathrm{Bi}+C_{\lambda 1}\right)\left(T_{f}-T_{0}\right)} \\
\frac{\partial t_{d}}{\partial \rho}=\frac{c z^{2}}{k} \tau_{d}
\end{gathered}
$$

The partial derivative of $\tau_{d} \mathrm{Bi}$ with respect to $\mathrm{Bi}$ is plotted in Figure 10. Since the value of $\partial\left(\tau_{d} \mathrm{Bi}\right) / \partial \mathrm{Bi}$ is always positive, $\partial t_{d} / \partial k$ is always negative. Therefore, as the thermal conductivity increases, the critical freezing time decreases nonlinearly. The summation of $C_{\lambda 2}$ and $C_{\eta}$ as a function of $\mathrm{Bi}$ is plotted in Figure 11. Since the summation is always negative, $\partial t_{d} / \partial c$ is always positive. Therefore, as the specific heat increases, the critical freezing time increases nonlinearly. According to Eqs. (22) and (23), as the latent heat or density increases, the critical freezing time increases linearly. 


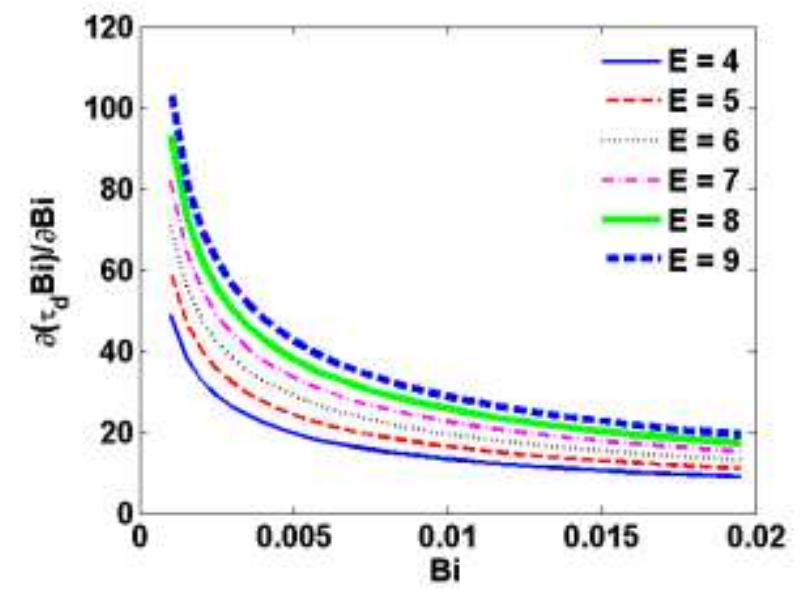

Figure 10. Partial derivative of $\tau_{d} \mathrm{Bi}$ with respect to $\mathrm{Bi}$.

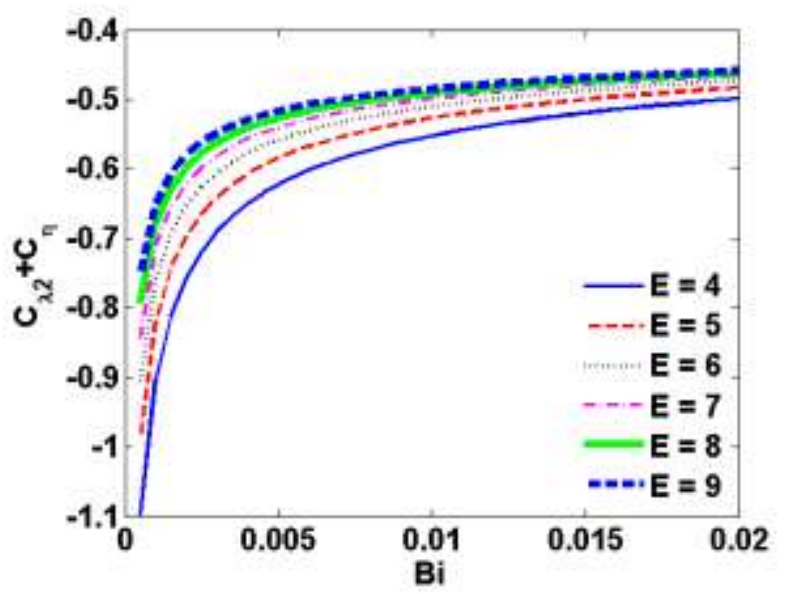

Figure 11. Summation of $C_{\lambda 2}$ and $C_{\eta}$ as a function of Bi.

\subsubsection{Paste solids loading}

Similar to the paste material, the change of paste solids loading will change all of the material properties. When paste material changes, the properties change discretely to other values. When paste solids loading changes, the properties changes continuously. Previous research has shown that paste density, specific heat, and latent heat can be computed by the law of mixtures. Also, paste thermal conductivity can be estimated using Eq. (11). Let the subscripts 
$c$ and $w$ denote ceramic and water, respectively, and the variable $v$ denote volume fraction. To study the effect of paste solids loading, Eq. (6) is differentiated with respect to ceramic volume fraction

$$
\begin{aligned}
\frac{\partial t_{d}}{\partial v_{c}}= & \frac{\partial t_{d}}{\partial k} \frac{\partial k}{\partial v_{c}}+\frac{\partial t_{d}}{\partial c} \frac{\partial c}{\partial v_{c}}+\frac{\partial t_{d}}{\partial L} \frac{\partial L}{\partial v_{c}}+\frac{\partial t_{d}}{\partial \rho} \frac{\partial \rho}{\partial v_{c}} \\
= & \frac{3\left(k_{c}-k_{w}\right)\left\{1+2\left\{\left[\left(3 v_{c}-1\right) k_{c}+\left(2-3 v_{c}\right) k_{w}\right]^{2}+8 k_{c} k_{w}\right\}^{-\frac{1}{2}}\right\}}{\frac{\partial t_{d}}{\partial k}} \\
& +\frac{\left(c_{c}-c_{w}\right) \rho_{c} \rho_{w}}{\left[\rho_{c} v_{c}+\rho_{w}\left(1-v_{c}\right)\right]^{2}} \frac{\partial t_{d}}{\partial c}+\frac{-\rho_{c} \rho_{w} L_{w}}{\left[\rho_{c} v_{c}+\rho_{w}\left(1-v_{c}\right)\right]^{2}} \frac{\partial t_{d}}{\partial L}+\left(\rho_{c}-\rho_{w}\right) \frac{\partial t_{d}}{\partial \rho}
\end{aligned}
$$

Typically, the ceramic particles have larger thermal conductivity, smaller specific heat, and larger density than water. In this case, the first three terms of Eq. (24) are negative, and the last term of Eq. (24) is positive. However, because of the large latent heat, the term $\frac{-\rho_{c} \rho_{w} L_{w}}{\left[\rho_{c} v_{c}+\rho_{w}\left(1-v_{c}\right)\right]^{2}} \frac{\partial t_{d}}{\partial L}$ dominates for the materials studied in this paper. In this case, as the paste solids loading increases, the critical freezing time decreases nonlinearly.

\subsubsection{Ambient temperature}

To study the effect of ambient temperature, Eq. (6) is differentiated with respect to ambient temperature. Ambient temperature only affects the non-dimensional latent heat. Therefore

$$
\frac{\partial t_{d}}{\partial T_{0}}=\frac{\rho c z^{2}}{k} \frac{\partial \tau_{d}}{\partial E} \frac{\partial E}{\partial T_{0}} \approx \frac{\rho L z^{2}}{k\left(T_{f}-T_{0}\right)^{2}} \frac{1}{\left(1+2 \mathrm{Bi}+C_{\lambda 1}\right)}
$$

All of the parameters in Eq. (25) are positive; therefore, the derivative is also positive. For the same paste and environmental conditions, as the ambient temperature increases, the critical freezing time increases nonlinearly. 


\subsubsection{Filament height}

To study the effect of filament height, Eq. (6) is differentiated with respect to filament height. Filament height appears in both the conversion from non-dimensional freezing time to dimensional freezing time and in the effective Biot number. Therefore

$$
\frac{\partial t_{d}}{\partial z}=\frac{\rho c}{k}\left(2 z \tau_{d}+z^{2} \frac{\partial \tau_{d}}{\partial \mathrm{Bi}} \frac{\partial \mathrm{Bi}}{\partial z}\right)=\frac{2 \rho c z}{k}\left(\tau_{d}+\mathrm{Bi} \frac{\partial \tau_{d}}{\partial \mathrm{Bi}}\right)=\frac{2 \rho c z}{k} \frac{\partial\left(\tau_{d} \mathrm{Bi}\right)}{\partial \mathrm{Bi}}
$$

As plotted in Figure 10, the partial derivative in Eq. (26) is always positive. Therefore, for the same paste and environmental conditions, as the filament height increases, the critical freezing time increases nonlinearly.

\subsubsection{Filament width}

To study the effect of filament width, Eq. (6) is differentiated with respect to the filament width. Filament width only affects the effective Biot number. Therefore

$$
\frac{\partial t_{d}}{\partial y}=\frac{\rho c z^{2}}{k} \frac{\partial \tau_{d}}{\partial \mathrm{Bi}} \frac{\partial \mathrm{Bi}}{\partial y}=-\frac{h \rho c z^{4}}{k^{2} y^{2}} \frac{\partial \tau_{d}}{\partial \mathrm{Bi}}
$$

As plotted in Figure 8, the term $\partial \tau_{d} / \partial \mathrm{Bi}$ is always negative. Therefore, this derivative is always positive. For the same paste and environmental conditions, as the filament width increases, the critical freezing time increases nonlinearly.

\section{Experimental Validation}

With the model derived in Section 2, the critical freezing time of a thin-wall part can be computed efficiently, and the error can be analyzed with the relationship derived in Section 3. 


\subsection{Experimental Setup and Paste Preparation}

The paste was a combination of $\mathrm{Al}_{2} \mathrm{O}_{3}$ powder (A-16SG, ALMATIS), DARVAN® C-N (Ammonium polymethacrylate, Vanderbilt Minerals LLC), METHOCEL F4M (Methylcellulose, Dow Chemical Company) and deionized water. The alumina powder was dispersed in water using DARVAN C and then ball-milled for approximately 15 hours to break up agglomerates and to produce a uniform mixture. METHOCEL was then added to the slurry at $70^{\circ} \mathrm{C}$ with mechanical stirring to increase paste viscosity and to assist in forming a stronger green body after drying. Finally, a vacuum mixer (Whip Mix, Model F) was used to remove air bubbles.

The FEF setup is comprised of three subsystems: a gantry controlled by a motion card (DELTA TAU Data Systems Inc.), extruders controlled by LabVIEW, and a freezer encompassing the entire machine. Figure 12 shows different parts of the FEF machine. The chamber temperature, also referred to as the ambient temperature, is reduced from room temperature using a liquid nitrogen tank connected to the chamber via tubing. A solenoid valve is used to maintain the ambient temperature at the desired value by connecting/disconnecting the flow of nitrogen into the chamber. To preclude freezing of the paste inside the syringe, a specially designed heating jacket with a controllable temperature was employed. Another heater surrounds the nozzle to prevent clogging as a result of freezing of the paste at the nozzle tip. The paste is extruded through a $610 \mu \mathrm{m}$ diameter nozzle and deposited on an aluminum substrate which is at ambient temperature (a thermocouple is utilized to measure this temperature as well). Two fans cause the air to flow around the part being fabricated with a speed of approximately 3 $\mathrm{m} / \mathrm{s}$ to enable forced convection. 


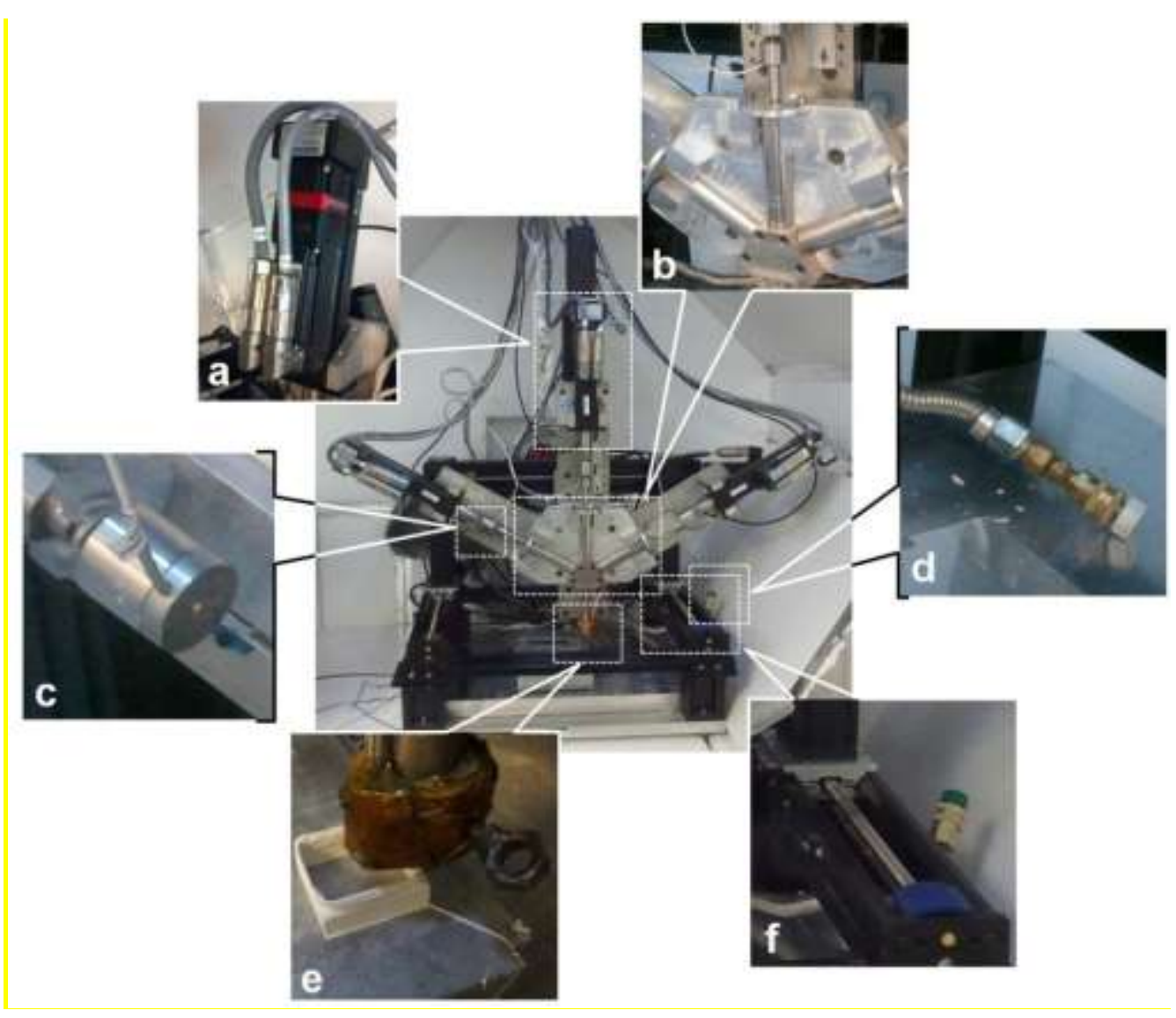

Figure 12. FEF machine with (a) servo motor (b) syringe housing (c) load cell (d) nitrogen flow outlet (e) nozzle and heater (f) gantry linear axis.

To validate the analytical results, thin-wall square parts were fabricated with different values of process parameters. If each layer has enough time to freeze, it can bear the weight of next layers and the part will stand. However, if each layer does not have enough time to freeze, the part will deform and possibly collapse. Table 2 shows the parameters affecting the critical time and their values during the experiments. 
Table 2. Constant and variable parameters used in experimental studies.

\begin{tabular}{cc}
\hline Constant Parameter & Value \\
\hline Syringe temperature $\left({ }^{\circ} \mathrm{C}\right)$ & 20 \\
Nozzle temperature $\left({ }^{\circ} \mathrm{C}\right)$ & 20 \\
Filament width $(\mu \mathrm{m})$ & 1010 \\
\hline Variable Parameter & Value \\
\hline Solids Loading $(\%$ Vol. $)$ & 45,60 \\
Convection condition & Forced, Natural \\
Ambient temperature $\left({ }^{\circ} \mathrm{C}\right)$ & $-10,-20$ \\
Filament height $(\mu \mathrm{m})$ & 300,500 \\
\hline
\end{tabular}

To obtain the critical freezing time experimentally for each set of parameters, various nozzle travel speeds and part side lengths were examined. Clearly, if the nozzle moves faster or the part has a shorter side length, the total time between layers will be smaller. In these experiments, two different nozzle travel speeds ( 12.7 and $21.2 \mathrm{~mm} / \mathrm{s}$ ) and 12 different part side lengths (17.8-68.6 $\mathrm{mm}$ ) were used to find critical freezing time experimentally and compare them with analytical predictions. In order to prove the experimental results are repeatable, two batches of paste were used in separate experimental runs for both groups.

\subsection{Possible Error Sources}

There are uncertainties in the values of some of the parameters which may affect the accuracy of the experimental results. The actual solids loadings of the pastes are not exactly equal to the desired values and were found to vary by approximately $3 \%$. Furthermore, the trace amount of binder and dispersant were neglected when calculating the thermal conductivity of the paste. The flow rate of the paste from the nozzle is not always constant during an experiment as a result of minor inconsistencies in the paste preparation process and the paste compressibility. This was found to cause approximately 5\% variation in filament width. Although a closed loop control system was employed to control the temperature inside the chamber, variations of 
approximately $\pm 1^{\circ} \mathrm{C}$ were observed. The air flow condition in the chamber can be very complex. Even when the fan is off, due to the flow of nitrogen and the machine motion, the part will not experience pure natural convection. When the fan is on, the inside of the part does not have exactly the same convection condition as the outside of the part, nor does it have pure natural convection. The effects of the various error sources on critical freezing time are discussed case by case in the next section.

\subsection{Experimental Results}

\subsubsection{Experiment group 1}

For experiment group1, the material was $45 \%$ solids loading alumina paste, the convection condition was forced, the ambient temperature was $-20^{\circ} \mathrm{C}$, the nozzle travel speed was 12.7 $\mathrm{mm} / \mathrm{s}$, and the filament height and width were 500 and $1010 \mu \mathrm{m}$, respectively. The estimated average thermal conductivity of this paste is $10.48 \mathrm{~W} / \mathrm{m} \cdot{ }^{\circ} \mathrm{C}$. With these experiment conditions, the predicted critical freezing time is $8.14 \mathrm{~s}$ and the corresponding critical part side length is 25.9 $\mathrm{mm}$. If the part has a larger size, it should stand (i.e., not deform or collapse). If the part has a smaller size, it is expected to deform or collapse. The results of experiment group 1 are given in Table 3, and example part photos are shown in Figure 13. When the total time between layers is close to the critical freezing time, a small change in the total time between layers may result in a dramatically different build, as seen in photos (a) and (b) in Figure 13. The critical freezing times measured for the pastes in batches \#1 and \#2 are 9.6 and $8.4 \mathrm{~s}$, respectively, and the errors between the predicted and measured results for batches \#1 and \#2 are $17.9 \%$ and $3.2 \%$, respectively. The effects of possible error sources are shown in Figure 14. Because the ambient temperature is $-20^{\circ} \mathrm{C}$, the effect of ambient temperature and convection coefficient error are not 
as significant as the effect of solids loading error. If the paste solids loading decreases to $40 \%$, the critical freezing time will increase approximately $25 \%$. If the ambient temperature increases to $-18{ }^{\circ} \mathrm{C}$ or the convection coefficient decreases $10 \%$, the critical freezing time will increase less than $10 \%$.

Table 3. Experimental and predicted results for experiment group 1.

\begin{tabular}{ccc}
\hline Side Length $(\mathrm{mm})$ & Experimental Result & Predicted Result \\
\hline & Paste batch \#1 \\
\hline 17.8 & Collapsed & Collapsed \\
22.9 & Collapsed & Collapsed \\
25.4 & Collapsed & Collapsed \\
27.9 & Deformed & Stood \\
33.0 & Stood & Stood \\
\hline \multicolumn{3}{c}{ Paste batch \#2 } \\
22.3 & Collapsed & Collapsed \\
25.9 & Collapsed & Collapsed \\
27.9 & Deformed & Collapsed \\
33.0 & Stood & Stood \\
\hline
\end{tabular}

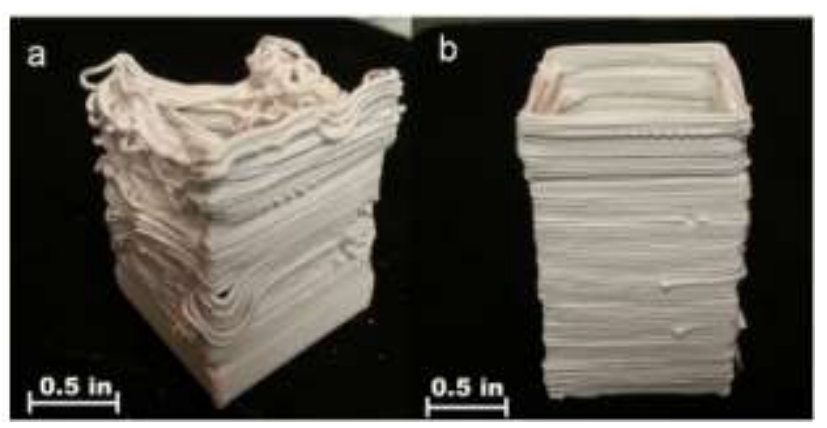

Figure 13. Parts fabricated in experiment group 1 using paste batch \#2 (a) $25.4 \mathrm{~mm}$ (b) 27.9 mm. 


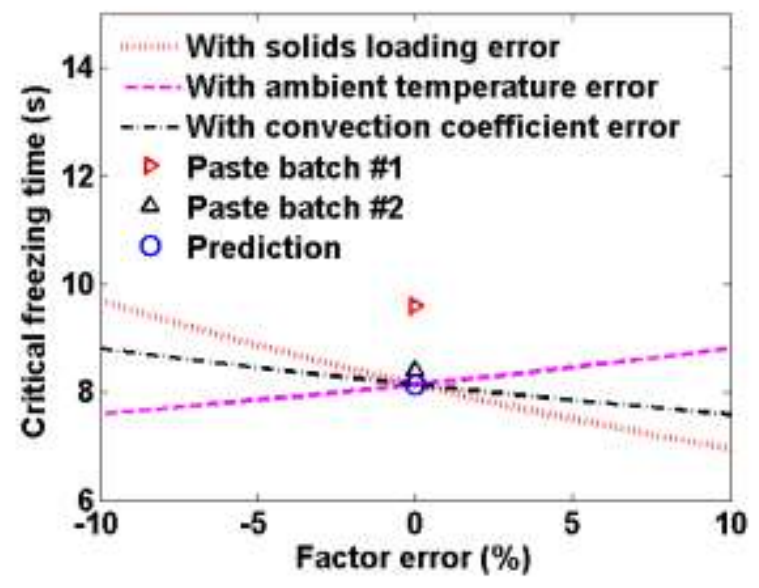

Figure 14. Predicted critical freezing time and effects of error sources for experiment group 1.

\subsubsection{Experiment group 2}

For experiment group 2 , the material was $60 \%$ solids loading alumina paste, the convection condition was natural, the ambient temperature was $-10{ }^{\circ} \mathrm{C}$, the nozzle travel speed was 21.2 $\mathrm{mm} / \mathrm{s}$, and the filament height and width were 300 and $1010 \mu \mathrm{m}$, respectively. The estimated average thermal conductivity of this paste is $18.81 \mathrm{~W} / \mathrm{m} \cdot{ }^{\circ} \mathrm{C}$. With these experiment conditions, the predicted critical freezing time is $11.16 \mathrm{~s}$ and the corresponding critical part side length is $59.1 \mathrm{~mm}$. The results of experiment group 2 are given in Table 4, and example part photos are shown in Figure 15. Although the freezing time and fabrication result are very different for each sample in Figures 13 and 15, the first 12-15 layers of each sample visually have good quality. This phenomenon can be explained by the work conducted by Li et al. (2014). When the paste is close to the substrate, conduction dominates the heat transfer process and the first several layers freeze very fast despite the convection condition and total time between layers. The lumped method presented in this paper mainly focuses on computing the critical freezing time, which can be used to predict whether parts with large numbers of layers can be successfully built. If a part 
has only a few layers, then the part may still be successfully built even if the total time between layers is shorter than the critical freezing time. In that case, the numerical simulation method presented by Li et al. (2014) is more suitable for computing the freezing time and determining if the part can be successfully built.

The critical freezing times measured for the pastes in batches \#1 and \#2 are 12.5 and $12.0 \mathrm{~s}$, respectively, and the errors between the predicted and measured results for batches \#1 and \#2 are $12.0 \%$ and $7.5 \%$, respectively. The effects of possible error sources are shown in Figure 16. The effect of solids loading error is still large. If the paste solids loading decreases to $55 \%$, then the critical freezing time will increase approximately $20 \%$. If the ambient temperature increases to $9{ }^{\circ} \mathrm{C}$, the critical freezing time will increase less than $10 \%$. The effect of convection coefficient becomes less significant since the parts fabricated in group 2 are subjected to natural convection instead of forced convection.

Table 4. Experimental and predicted results for experiment group 2.

\begin{tabular}{ccc}
\hline Side Length $(\mathrm{mm})$ & Experimental Result & Predicted Result \\
\hline & Paste batch \#1 \\
\hline 55.9 & Collapsed & Collapsed \\
61.0 & Collapsed & Stood \\
63.5 & Deformed & Stood \\
68.6 & Stood & Stood \\
76.2 & Stood & Stood \\
\hline \multicolumn{3}{c}{ Paste batch \#2 } \\
\hline 65.9 & Collapsed & Collapsed \\
66.0 & Collapsed & Stood \\
68.6 & Stood & Stood \\
\hline
\end{tabular}




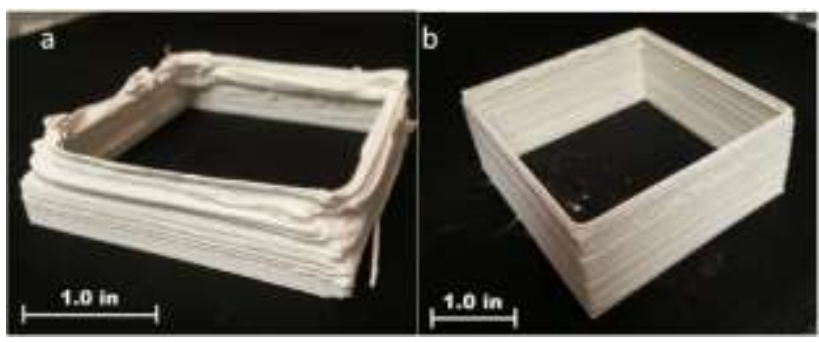

Figure 15. Parts fabricated in experiment group 2 using paste batch \#2 (a) $61.0 \mathrm{~mm}$ (b) 66.0 mm.

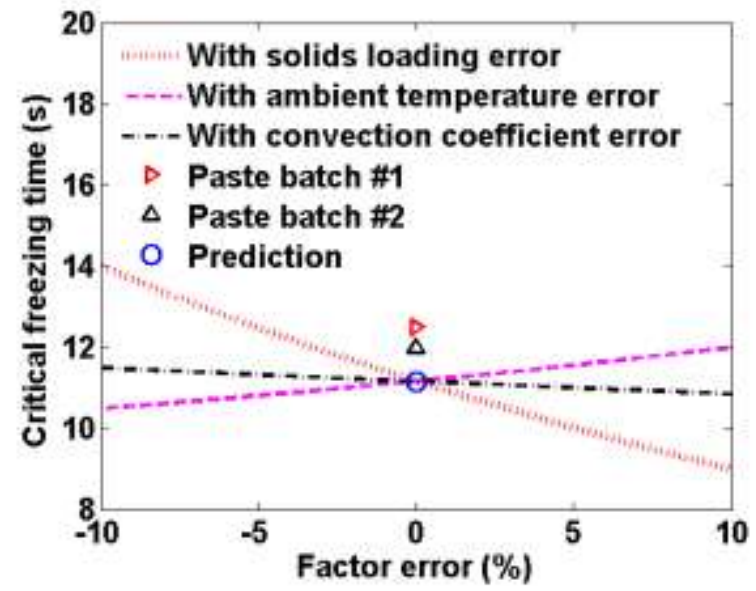

Figure 16. Predicted critical freezing time and effects of error sources for experiment group 2.

\section{Summary and Conclusions}

An analytical freezing time model for thin-wall parts was developed using a lumped method to gain more physical understanding about the freezing process of aqueous-based pastes extruded in a freezing environment. This method can be used to predict the freezing time of thin-wall parts with large numbers of layers. For parts with small numbers of layers, a detailed numerical simulation is required to compute the freezing time. The major assumption in the lumped method is that the temperature gradient is negligible inside a single paste filament. 
Two non-dimensional factors, the non-dimensional latent heat and the effective Biot number, were shown to be the main variables affecting the non-dimensional critical freezing time. The two non-dimensional factors are affected by six factors, i.e., convection coefficient, paste material, paste solids loading, ambient temperature, filament height, and filament width. The effects of all of the non-dimensional and dimensional factors on the critical freezing time were studied and the results are summarized in Table 5.

Table 5. Factors and their effects on critical freezing time

\begin{tabular}{ll}
\hline Factor & $\begin{array}{l}\text { How critical freezing } \\
\text { time changes when } \\
\text { factor value increases }\end{array}$ \\
\hline \multicolumn{2}{c}{ Non-dimensional factors } \\
\hline $\begin{array}{l}\text { Non-dimensional latent heat } \\
\text { Effective Biot number }\end{array}$ & $\begin{array}{l}\text { Increases nonlinearly } \\
\text { Decreases nonlinearly }\end{array}$ \\
\hline \multicolumn{2}{c}{ Dimensional factors } \\
\hline Convection coefficient & Decreases nonlinearly \\
Ambient temperature & Increases nonlinearly \\
Filament height & Increases nonlinearly \\
Filament width & Increases nonlinearly \\
Paste solids loading & Decreases nonlinearly \\
Paste material properties & \\
\multicolumn{2}{c}{ Thermal conductivity } \\
Specific heat & Decreases nonlinearly \\
Latent heat & Increases nonlinearly \\
Density & Increases linearly \\
\hline
\end{tabular}

Experiments were conducted to validate the analytical freezing time model using different pastes and extrusion parameters. The measured critical freezing times have good agreement with the predicted critical freezing times using the lumped method, as the average error is approximately $10 \%$ and the maximum error is $17.9 \%$. The errors are mainly due to the uncertainties in the ambient temperature, ambient air flow, and paste solids loading. 


\section{Acknowledgments}

This work was supported by the National Science Foundation (CMMI 0856419) and the Center for Aerospace Manufacturing Technologies at the Missouri University of Science and Technology.

\section{Appendix}

The explicit form of non-dimensional enthalpy history $\boldsymbol{\eta}(\tau)$ for a thin wall can be solved from the matrix form of the non-dimensional energy governing equation

$$
\left[\begin{array}{c}
\boldsymbol{\eta}_{s}^{\prime}(\tau) \\
\boldsymbol{\eta}_{m}^{\prime}(\tau) \\
\boldsymbol{\eta}_{l}^{\prime}(\tau)
\end{array}\right]=\left[\begin{array}{ccc}
\mathbf{M}_{s} & \mathbf{O} & \mathbf{O} \\
\mathbf{U} & \mathbf{O} & \mathbf{D} \\
\mathbf{O} & \mathbf{O} & \mathbf{M}_{l}
\end{array}\right]\left[\begin{array}{l}
\boldsymbol{\eta}_{s}(\tau) \\
\boldsymbol{\eta}_{m}(\tau) \\
\boldsymbol{\eta}_{l}(\tau)
\end{array}\right]+\left[\begin{array}{l}
\boldsymbol{\beta}_{s} \\
\boldsymbol{\beta}_{m} \\
\boldsymbol{\beta}_{l}
\end{array}\right]
$$

where

$$
\mathbf{M}_{s}=\left[\begin{array}{ccccc}
-(3+2 \mathrm{Bi}) & 1 & 0 & \cdots & 0 \\
\ddots & \ddots & \ddots & \ddots & \vdots \\
\vdots & 1 & -2(1+\mathrm{Bi}) & 1 & \vdots \\
\vdots & \ddots & \ddots & \ddots & \ddots \\
0 & \cdots & 0 & 1 & -2(1+\mathrm{Bi})
\end{array}\right]
$$

$$
\mathbf{M}_{l}=\left[\begin{array}{ccccc}
-2(1+\mathrm{Bi}) & 1 & 0 & \cdots & 0 \\
\ddots & \ddots & \ddots & \ddots & \vdots \\
\vdots & 1 & -2(1+\mathrm{Bi}) & 1 & \vdots \\
\vdots & \ddots & \ddots & \ddots & \ddots \\
0 & \cdots & 0 & 1 & -\left(1+2 \mathrm{Bi}+\mathrm{Bi}_{z}\right)
\end{array}\right]
$$

$$
\mathbf{U}=\left[\begin{array}{cccc}
0 & \cdots & 0 & 1 \\
\vdots & \therefore & 0 & 0 \\
\vdots & \therefore & . & \vdots \\
0 & \cdots & \cdots & 0
\end{array}\right]
$$




$$
\begin{aligned}
& \mathbf{D}=\left[\begin{array}{cccc}
0 & \cdots & \cdots & 0 \\
\vdots & . & . & \vdots \\
0 & 0 & . & \vdots \\
1 & 0 & \cdots & 0
\end{array}\right] \\
& \boldsymbol{\beta}_{s}=\left[\begin{array}{llll}
0 & \cdots & 0 & 1
\end{array}\right]^{T} \\
& \boldsymbol{\beta}_{m}=\left[\begin{array}{lllll}
-(1+2 \mathrm{Bi}) & -2 \mathrm{Bi} & \cdots & -2 \mathrm{Bi} & -(1+2 \mathrm{Bi}+E)
\end{array}\right]^{T} \\
& \boldsymbol{\beta}_{l}=\left[\begin{array}{lllll}
(1+2 \mathrm{Bi} E+E) & 2 \mathrm{Bi} E & \cdots & 2 \mathrm{Bi} E & \left(2 \mathrm{Bi}+\mathrm{Bi}_{z}\right) E
\end{array}\right]^{T}
\end{aligned}
$$

and $\boldsymbol{\eta}_{*}^{\prime}(\tau)$ is the derivative of $\boldsymbol{\eta}_{*}(\tau)$ with respect to $\tau$, where the subscript $*$ is $s$ for the solid region, $m$ for the mushy region, and $l$ for the liquid region. The solution for the solid and liquid regions is

$$
\boldsymbol{\eta}_{*}(\tau)=\mathbf{Q}_{*}\left[\mathbf{P}_{*} \boldsymbol{\eta}_{*}(0) \circ \exp \left(\boldsymbol{\lambda}_{*} \tau\right)\right]+\mathbf{Q}_{*}\left\{\mathbf{P}_{*} \circ\left\{\left\{\left[\exp \left(\boldsymbol{\lambda}_{*} \tau\right)-1\right] \circ \frac{1}{\boldsymbol{\lambda}_{*}}\right\} \mathbf{v}^{T}\right\}\right\} \boldsymbol{\beta}_{*}
$$

where the symbol ${ }^{\circ}$ denotes element-wise product, $\lambda$ is the eigenvalue vector of $\mathbf{M}, \mathbf{Q}$ is the corresponding eigenvector matrix, $\mathbf{P}=\mathbf{Q}^{-1}$, and

$$
\mathbf{v}=\left[\begin{array}{lllll}
1 & \cdots & 1 & \cdots & 1
\end{array}\right]^{T}
$$

The solution for the mushy region is

$$
\boldsymbol{\eta}_{m}(\tau)=\boldsymbol{\eta}_{m}(0)+\boldsymbol{\beta}_{m} \tau+\boldsymbol{\varphi}_{m}(\tau)
$$

where

$$
\begin{aligned}
& \boldsymbol{\varphi}_{m}(\tau)=\left[\int_{0}^{\tau} \eta_{s, N_{s}}(\varepsilon) d \varepsilon \quad 0 \quad \cdots \quad c \quad c \quad \cdots \quad 0 \quad \int_{0}^{\tau} \eta_{l, 1}(\varepsilon) d \varepsilon\right]^{T} \\
& \int_{0}^{\tau} \boldsymbol{\eta}_{*}(\varepsilon) d \varepsilon=\mathbf{Q}_{*}\left\{\mathbf{P}_{*} \boldsymbol{\eta}_{*}(0) \circ\left[\exp \left(\boldsymbol{\lambda}_{*} \tau\right)-1\right] \circ \frac{1}{\boldsymbol{\lambda}_{*}}\right\} \\
& +\mathbf{Q}_{*}\left\{\mathbf{P}_{*} \circ\left\{\left\{\left[\exp \left(\boldsymbol{\lambda}_{*} \tau\right)-\boldsymbol{\lambda}_{*} \tau-1\right] \circ \frac{1}{\boldsymbol{\lambda}_{*} \circ \boldsymbol{\lambda}_{*}}\right\} \mathbf{v}^{T}\right\}\right\} \boldsymbol{\beta}_{*}
\end{aligned}
$$


For the solid region, the elements of the corresponding matrices and vectors are (Yueh, 2005)

$$
\begin{gathered}
\lambda_{s, i}=-2\left[1+\mathrm{Bi}-\cos \left(\frac{2 i \pi}{2 N_{s}+1}\right)\right] \\
Q_{s, i j}=\sin \left[\frac{(2 i-1) j \pi}{2 N_{s}+1}\right] \\
P_{s, i j}=\frac{4}{2 N_{s}+1} Q_{s, j i}=\frac{4}{2 N_{s}+1} \sin \left[\frac{i(2 j-1) \pi}{2 N_{s}+1}\right]
\end{gathered}
$$

In most of the cases studied, $\mathrm{Bi}_{z}$ is typically smaller than 0.01 ; therefore, $\mathrm{Bi}_{z} \approx 0$. The physical meaning of this approximation is that the top of the liquid region is modeled as an insulated surface. In this case, Eq. (A3) becomes

$$
\mathbf{M}_{l}=\left[\begin{array}{ccccc}
-2(1+\mathrm{Bi}) & 1 & 0 & \cdots & 0 \\
\ddots & \ddots & \ddots & \ddots & \vdots \\
\vdots & 1 & -2(1+\mathrm{Bi}) & 1 & \vdots \\
\vdots & \ddots & \ddots & \ddots & \ddots \\
0 & \cdots & 0 & 1 & -(1+2 \mathrm{Bi})
\end{array}\right]
$$

and the elements of the corresponding matrices and vectors for the liquid region are (Yueh, 2005)

$$
\begin{gathered}
\lambda_{l, i}=-2\left[1+\mathrm{Bi}-\cos \left(\frac{2 i-1}{2 N_{l}+1} \pi\right)\right] \\
Q_{l, i j}=\sin \left[\frac{i(2 j-1) \pi}{2 N_{l}+1}\right] \\
P_{l, i j}=\frac{4}{2 N_{l}+1} Q_{l, j i}=\frac{4}{2 N_{l}+1} \sin \left[\frac{(2 i-1) j \pi}{2 N_{l}+1}\right]
\end{gathered}
$$

When a new layer is deposited, the freezing time of the layer is composed of two parts. The first part is the time spent for the liquid region to transform into a mushy region. The second part is the time spent for the mushy region to transform into a solid region. Due to the fact that the 
latent heat of paste is much larger than the specific heat of paste and the initial paste temperature is very close to the freezing temperature, the liquid region transforms into a mushy region much faster than the mushy region transforms into a solid region. Therefore, the freezing time is approximated by the summation of the non-dimensional times required for each layer in the mushy region to transform a into solid layer

$$
t_{f}=\frac{\rho c z^{2}}{k} \sum_{n=1}^{N_{m}} \tau_{f, n}
$$

where $\tau_{f, n}$ is the non-dimensional time for the $n^{\text {th }}$ mushy layer to transform into a solid layer. This non-dimensional time is obtained by solving

$$
\eta_{m, n}\left(\tau_{f, n}\right)=1 \quad\left(n=1,2, \ldots, N_{m}\right)
$$

In this case, the liquid region does not need to be considered and Eq. (A1) becomes

$$
\left[\begin{array}{c}
\boldsymbol{\eta}_{s}^{\prime}(\tau) \\
\boldsymbol{\eta}_{m}^{\prime}(\tau)
\end{array}\right]=\left[\begin{array}{cc}
\mathbf{M}_{s} & \mathbf{O} \\
\mathbf{U} & \mathbf{O}
\end{array}\right]\left[\begin{array}{l}
\boldsymbol{\eta}_{s}(\tau) \\
\boldsymbol{\eta}_{m}(\tau)
\end{array}\right]+\left[\begin{array}{l}
\boldsymbol{\beta}_{s} \\
\boldsymbol{\beta}_{m}
\end{array}\right]
$$

This change means there is no liquid region and the top surface of the mushy region is directly exposed to the ambient. Therefore, the terms in Eq. (A11) become

$$
\begin{gathered}
\boldsymbol{\beta}_{m}=\left[\begin{array}{llllll}
-(1+2 \mathrm{Bi}) & -2 \mathrm{Bi} & \cdots & -2 \mathrm{Bi} & -\left(1+2 \mathrm{Bi}+\mathrm{Bi}_{z}\right)
\end{array}\right]^{T} \\
\boldsymbol{\varphi}_{m}(\tau)=\left[\begin{array}{lllllll}
\int_{0}^{\tau} \eta_{s, N_{s}}(\varepsilon) d \varepsilon & 0 & \cdots & 0 & \cdots & 0
\end{array}\right]^{T}
\end{gathered}
$$

Substituting Eqs. (A14)-(A16) into Eq. (A11)

$$
\begin{aligned}
\eta_{m, 1}(\tau) & =\eta_{m, 1}(0)-(1+2 \mathrm{Bi}) \tau+\frac{4}{2 N_{s}+1} \sum_{i=1}^{N_{s}}\left\{\frac{\exp \left(\lambda_{s, i} \tau\right)-\lambda_{s, i} \tau-1}{\lambda_{s, i}^{2}} \sin ^{2}\left[\frac{\left(2 N_{s}-1\right) i \pi}{2 N_{s}+1}\right]\right\} \\
& +\frac{4}{2 N_{s}+1} \sum_{i=1}^{N_{s}}\left\{\frac{\exp \left(\lambda_{s, i} \tau\right)-1}{\lambda_{s, i}} \sin \left[\frac{\left(2 N_{s}-1\right) i \pi}{2 N_{s}+1}\right] \sum_{j=1}^{N_{s}}\left\{\sin \left[\frac{i(2 j-1) \pi}{2 N_{s}+1}\right] \eta_{s, j}(0)\right\}\right\}
\end{aligned}
$$

When $\tau \gg>0$, the terms $\exp \left(\lambda_{n} \tau\right)$ are negligible for $n>1$. Therefore, Eq. (A22) becomes 


$$
\begin{aligned}
& \frac{4}{2 N_{s}+1}\left\{\frac{1}{\lambda_{s, 1}^{2}} \sin ^{2}\left(\frac{2 \pi}{2 N_{s}+1}\right)+\frac{1}{\lambda_{s, 1}} \sin \left(\frac{2 \pi}{2 N_{s}+1}\right) \sum_{j=1}^{N_{s}}\left\{\sin \left[\frac{(2 j-1) \pi}{2 N_{s}+1}\right] \eta_{s, j}(0)\right\}\right\} \exp \left(\lambda_{s, 1} \tau\right) \\
& =\left\{(1+2 \mathrm{Bi})+\frac{4}{2 N_{s}+1} \sum_{i=1}^{N_{s}}\left[\frac{1}{\lambda_{s, i}} \sin ^{2}\left(\frac{2 i \pi}{2 N_{s}+1}\right)\right]\right\} \tau \\
& +\eta_{m, 1}(\tau)-\eta_{m, 1}(0)+\frac{4}{2 N_{s}+1} \sum_{i=1}^{N_{s}}\left[\frac{1}{\lambda_{s, i}^{2}} \sin ^{2}\left(\frac{2 i \pi}{2 N_{s}+1}\right)\right] \\
& +\frac{4}{2 N_{s}+1} \sum_{i=1}^{N_{s}}\left\{\frac{1}{\lambda_{s, i}} \sin \left[\frac{\left(2 N_{s}-1\right) i \pi}{2 N_{s}+1}\right] \sum_{j=1}^{N_{s}}\left\{\sin \left[\frac{i(2 j-1) \pi}{2 N_{s}+1}\right] \eta_{s, j}(0)\right\}\right\}
\end{aligned}
$$

To prevent the part from deforming or collapsing during the fabrication process, the new layer should only be deposited when the previous layers are frozen. In that case, the mushy region typically only consists of one layer. Solving Eq. (A27), the non-dimensional freezing time is

$$
\tau_{f}=-\frac{W\left[-\frac{\lambda_{s, 1}}{\alpha_{s}} \exp \left(-\frac{\gamma_{s}}{\alpha_{s}} \lambda_{s, 1}\right)\right]}{\lambda_{s, 1}}-\frac{\gamma_{s}}{\alpha_{s}}
$$

where $W$ is the principal branch of Lambert $\mathrm{W}$ function and

$$
\begin{gathered}
\alpha_{s}=\frac{-\beta_{m, 1}+\theta_{s}}{\omega_{s}} \\
\gamma_{s}=\frac{1-\eta_{m, 1}(0)+\psi_{s}}{\omega_{s}}
\end{gathered}
$$

$$
\begin{gathered}
\omega_{s}=\frac{4}{2 N_{s}+1}\left\{\frac{1}{\lambda_{s, 1}^{2}} \sin ^{2}\left(\frac{2 \pi}{2 N_{s}+1}\right)+\frac{1}{\lambda_{s, 1}} \sin \left(\frac{2 \pi}{2 N_{s}+1}\right) \sum_{j=1}^{N_{s}}\left\{\sin \left[\frac{(2 j-1) \pi}{2 N_{s}+1}\right] \eta_{s, j}(0)\right\}\right\} \\
\theta_{s}=\frac{4}{2 N_{s}+1} \sum_{i=1}^{N_{s}}\left[\frac{1}{\lambda_{s, i}} \sin ^{2}\left(\frac{2 i \pi}{2 N_{s}+1}\right)\right] \\
\psi_{s}=\frac{4}{2 N_{s}+1} \sum_{i=1}^{N_{s}}\left[\frac{1}{\lambda_{s, i}^{2}} \sin ^{2}\left(\frac{2 i \pi}{2 N_{s}+1}\right)\right] \\
+\frac{4}{2 N_{s}+1} \sum_{i=1}^{N_{s}}\left\{\frac{1}{\lambda_{s, i}} \sin \left[\frac{\left(2 N_{s}-1\right) i \pi}{2 N_{s}+1}\right] \sum_{j=1}^{N_{s}}\left\{\sin \left[\frac{i(2 j-1) \pi}{2 N_{s}+1}\right] \eta_{s, j}(0)\right\}\right\}
\end{gathered}
$$


In the cases studied in this paper, $W\left[-\frac{\lambda_{s, 1}}{\alpha_{s}} \exp \left(-\frac{\gamma_{s}}{\alpha_{s}} \lambda_{s, 1}\right)\right]$ is small relative to the term $\gamma_{s} \lambda_{s, 1} / \alpha_{s}$ and, thus, may be neglected. In this case, Eq. (A28) becomes

$$
\tau_{f}=-\frac{1-\eta_{m, 1}(0)+\psi_{s}}{\theta_{s}-\beta_{m, 1}}
$$

Similar to the approximation in the liquid region, $\mathrm{Bi}_{z}$ is negligible in the cases studied in this paper. Letting the subscript $d$ denote steady state and noting $\eta_{m, 1}(0) \approx 1+E$, the steady-state freezing time is

$$
\tau_{d}=\frac{E-C_{\lambda 2}-C_{\eta}}{1+2 \mathrm{Bi}+C_{\lambda 1}}
$$

where

$$
\begin{gathered}
C_{\lambda 1}(\mathrm{Bi})=\lim _{N_{s} \rightarrow \infty}\left\{\frac{4}{2 N_{s}+1} \sum_{i=1}^{N_{s}}\left[\frac{1}{\lambda_{s, i}} \sin ^{2}\left(\frac{2 i \pi}{2 N_{s}+1}\right)\right]\right\} \\
C_{\lambda 2}(\mathrm{Bi})=\lim _{N_{s} \rightarrow \infty}\left\{\frac{4}{2 N_{s}+1} \sum_{i=1}^{N_{s}}\left[\frac{1}{\lambda_{s, i}^{2}} \sin ^{2}\left(\frac{2 i \pi}{2 N_{s}+1}\right)\right]\right\}=\frac{d C_{\lambda 1}}{2 d \mathrm{Bi}} \\
C_{\eta}\left(\mathrm{Bi}, \tau_{r}\right)=\lim _{N_{s} \rightarrow \infty}\left\{\frac{4}{2 N_{s}+1} \sum_{i=1}^{N_{s}}\left\{\frac{1}{\lambda_{s, i}} \sin \left[\frac{\left(2 N_{s}-1\right) i \pi}{2 N_{s}+1}\right] \sum_{j=1}^{N_{s}}\left\{\sin \left[\frac{i(2 j-1) \pi}{2 N_{s}+1}\right] \eta_{s, j}(0)\right\}\right\}\right\}
\end{gathered}
$$

and the subscript $r$ denotes the total time between layers (i.e., the summation of the deposition time for the current layer and the dwell time between the current and next layers).

Unlike Eqs. (A36) and (A37), Eq. (A38) cannot be simply approximated by the sum of a finite number of its initial terms, it needs to be solved iteratively. When the steady state occurs, $C_{\eta}$ must be constant, which requires 


$$
\begin{aligned}
& \frac{4}{2 N_{s}+1} \sum_{i=1}^{N_{s}}\left\{\frac{1}{\lambda_{s, i}} \sin \left[\frac{\left(2 N_{s}-1\right) i \pi}{2 N_{s}+1}\right] \sum_{j=1}^{N_{s}}\left\{\sin \left[\frac{i(2 j-1) \pi}{2 N_{s}+1}\right] \eta_{s, j}(0)\right\}\right\} \\
= & \frac{4}{2\left(N_{s}+1\right)+1} \sum_{i=1}^{N_{s}+1}\left\{\frac{1}{\lambda_{s, i}} \sin \left\{\frac{\left[2\left(N_{s}+1\right)-1\right] i \pi}{2\left(N_{s}+1\right)+1}\right\} \sum_{j=1}^{N_{s}+1}\left\{\sin \left[\frac{i(2 j-1) \pi}{2\left(N_{s}+1\right)+1}\right] \eta_{s, j}\left(\tau_{r}\right)\right\}\right\}
\end{aligned}
$$

where

$$
\begin{aligned}
\boldsymbol{\eta}_{s, N_{s}}\left(\tau_{r}\right)=\boldsymbol{\eta}_{s, N_{s}+1}(0)= & \frac{4}{2\left(N_{s}+1\right)+1}\left\{\mathbf{Q}_{s}\left\{\mathbf{Q}_{s}^{T}\left[\begin{array}{c}
\left.\boldsymbol{\eta}_{s, N_{s}}\left(\tau_{d}\right)\right] \\
1
\end{array}\right] \circ \exp \left[\boldsymbol{\lambda}_{s}\left(\tau_{r}-\tau_{d}\right)\right]\right\}\right\} \\
\boldsymbol{\eta}_{s, N_{s}}\left(\tau_{d}\right) & =\frac{4}{2 N_{s}+1} \mathbf{Q}_{s}\left[\mathbf{Q}_{s}^{T} \boldsymbol{\eta}_{s, N_{s}}(0) \circ \exp \left(\boldsymbol{\lambda}_{s} \tau_{d}\right)\right] \\
& +\frac{4}{2 N_{s}+1} \mathbf{Q}_{s}\left\{\mathbf{Q}_{s}^{T} \circ\left\{\left\{\left[\exp \left(\boldsymbol{\lambda}_{s} \tau_{d}\right)-1\right] \circ \frac{1}{\boldsymbol{\lambda}_{s}}\right\} \mathbf{v}_{s}^{T}\right\}\right\} \boldsymbol{\beta}_{s}
\end{aligned}
$$

Note that the steady-state freezing time is a function of the total time between layers. Also recall that the critical freezing time is defined as the time when the steady-state freezing time is equal to the total time between layers. When the critical freezing time occurs, Eqs. (A40) and (A41) are combined and

$$
\boldsymbol{\eta}_{s}\left(\tau_{d}\right)=\left[\begin{array}{c}
\frac{4}{2 N_{s}+1} \mathbf{Q}_{s}\left[\mathbf{Q}_{s}^{T} \boldsymbol{\eta}_{s}(0) \circ \exp \left(\boldsymbol{\lambda}_{s} \tau_{d}\right)\right] \\
+\frac{4}{2 N_{s}+1} \mathbf{Q}_{s}\left\{\mathbf{Q}_{s}^{T} \circ\left\{\left\{\left[\exp \left(\boldsymbol{\lambda}_{s} \tau_{d}\right)-1\right] \circ \frac{1}{\boldsymbol{\lambda}_{s}}\right\} \mathbf{v}_{s}^{T}\right\}\right\} \boldsymbol{\beta}_{s} \\
1
\end{array}\right]
$$

Substituting Eq. (A42) into Eq. (A39), Eq. (A38) can be solved iteratively. Thus, the nondimensional critical freezing time in Eq. (A35) (i.e., Eq. (6)) is obtained iteratively. 


\section{References}

Brailsford, A. D., and Major, K. G., 1964, The Thermal Conductivity of Aggregates of Several

Phases, Including Porous Materials, British Journal of Applied Physics, 15(3), pp 313-319.

Cengel, Y. A., 2002, Heat Transfer, a Practical Approach, WCB/McGraw-Hill, New York.

Denlinger, E. R., Irwin, J., and Michaleris, P., 2014, Thermomechanical Modeling of Additive

Manufacturing Large Parts, ASME Journal of Manufacturing Science and Engineering, 136(6), p. 061007.

Huang, T., Mason, M. S., Hilmas, G. E., and Leu, M. C., 2006, Freeze-Form Extrusion

Fabrication of Ceramic Parts, Virtual and Physical Prototyping, 1(2), pp. 93-100.

Huang, Y., Leu, M. C., Mazumder, J., and Donmez, A., 2015, Additive Manufacturing: Current

State, Future Potential, Gaps and Needs, and Recommendations, ASME Journal of Manufacturing Science and Engineering, 137(1), p. 014001.

Landauer, R., 1952, The Electrical Resistance of Binary Metallic Mixtures, Journal of Applied Physics, 23, pp. 779-784.

Li, M., Landers, R. G., and Leu, M. C., 2014, Modeling, Analysis, and Simulation of Paste Freezing in Freeze-Form Extrusion Fabrication of Thin-Wall Parts, ASME Journal of Manufacturing Science and Engineering, 136(6), p. 061003.

Michaleris, P., 2014, Modeling Metal Deposition in Heat Transfer Analyses of Additive Manufacturing Processes, Finite Elements in Analysis and Design, 86, pp. 51-60.

Tapia, G., and Elwany, A., 2014, A Review on Process Monitoring and Control in Metal-Based Additive Manufacturing, ASME Journal of Manufacturing Science and Engineering, 136(6), p. 060801. 
Yueh, W. C., 2005, Eigenvalues of Several Tridiagonal Matrices, Applied Mathematics E-Notes, 5, pp. 66-74.

Zeng, K., Pal, D., and Stucker, B., 2012, A Review of Thermal Analysis Methods in Laser Sintering and Selective Laser Melting, Solid Freeform Fabrication Symposium, Austin, TX, pp. 796-814. 